\title{
Effective Stratification for Pseudoadiabatic Ascent
}

\author{
JOHN W. NIELSEN-GAMMON \\ Department of Atmospheric Sciences, Texas A\&M University, College Station, Texas \\ DANIEL KeYSER \\ Department of Earth and Atmospheric Sciences, The University at Albany, State University of New York, \\ Albany, New York
}

24 August 1999 and 15 December 1999

\section{ABSTRACT}

\begin{abstract}
Effective stratification can be interpreted as the resistance to upward motion of saturated air parcels experiencing condensation. Previously published expressions for effective stratification conflict with each other, and the most widely distributed expression contains an $O(1)$ error. A derivation of effective stratification is presented that exposes its physical interpretation and that reveals the origin of the flaw in the incorrect derivation.
\end{abstract}

\section{Introduction}

The impact of latent heat release during stable moist ascent on the dynamics of atmospheric circulation systems can be assessed in one of two ways. In the first method, here called the external approach, latent heat release is regarded as an external forcing mechanism that alters the thermodynamic profile of the atmosphere and drives or helps to drive the vertical circulation. The second method, here called the stratification approach, explicitly treats the latent heat release as being proportional to the upward motion, so that this process is manifested as a modification to the stratification in the vertical advection term of the thermodynamic equation.

Each method is suited to a particular set of applications. The external approach is consistent with the quasigeostrophic system, which treats stratification as horizontally uniform, and is suitable when the latent heat release is a product of cumulus convection rather than explicitly resolved vertical motion. The stratification approach would seem more desirable when studying the dynamics of resolved large-scale ascent, because it constrains the latent heat release to be consistent with the diagnosed vertical motion and conforms with the idea that condensation is a consequence of vertical motion rather than a cause of it. In practice, the stratification approach has tended to be preferred by theoreticians (e.g., Emanuel 1985; Emanuel et al. 1987; Xu 1989;

Corresponding author address: Dr. John W. Nielsen-Gammon, MS 3150, Dept. of Atmospheric Sciences, Texas A\&M University, College Station, TX 77843-3150.

E-mail: n-g@tamu.edu
Whitaker and Davis 1994) and the external approach has tended to be preferred by diagnosticians (e.g., Kuo et al. 1991; Pauley and Nieman 1992), although a few diagnostic studies do incorporate latent heating into the vertical motion term (e.g., Hirschberg and Fritsch 1991; Lagouvardos et al. 1993). Diagnostic studies of other processes, such as frontogenesis, often follow the external approach by treating adiabatic cooling and diabatic heating separately (e.g., Orlanski et al. 1985) or by neglecting diabatic heating entirely (e.g., Schultz and Steenburgh 1999).

One practical difficulty in applying the stratification approach to an omega equation is that, in general, the stratification must not be so weak or unstable that the equation ceases to be elliptic. When the stratification is unstable and convection therefore is possible, the convective heating would need to be specified separately as an external forcing. This separation of latent heat release is consistent with the idea that balanced vertical motion [in the sense of Davis et al. (1996)] is diagnosable from the larger-scale flow, while explicit convective-scale vertical motion is not.

While the stratification approach perhaps should be more widely used, there is an inconsistency in published expressions of effective stratification, with the competing formulations differing by about a factor of 2 for common lower-tropospheric thermodynamic conditions. Thus, it is not clear, for those who desire to adopt the stratification approach, which effective stratification to use. The problem is analogous to the discrepancies in expressions for the moist Brunt-Väisälä frequency discussed by Durran and Klemp (1982). The purpose of this note is to derive and present an accurate expression 
for effective stratification (using an approach that emphasizes its physical interpretation), and to identify the source and nature of the error in alternative expressions that appear in textbooks such as Holton (1992).

\section{Derivation}

A valid expression for effective stratification is given in Emanuel et al. (1987). Presented below is an equivalent derivation that facilitates comparison with other derivations. For simplicity we consider only liquid-vapor phase transitions.

The starting point for this derivation is the existence of an equivalent potential temperature for saturated air $\left(\theta_{\mathrm{es}}\right)$ such that for an air parcel undergoing saturated ascent

$$
\frac{d s}{d t}=c_{p} \frac{d \ln \theta_{\mathrm{es}}}{d t}=0,
$$

where $s$ is the specific entropy (per unit mass of dry air) and $c_{p}$ is the heat capacity at constant pressure. With the standard thermodynamic assumptions outlined in Hauf and Höller (1987, pp. 2889-2890) [see also Iribarne and Godson (1981, pp. 82-84), Bohren and Albrecht (1998, pp. 287-288), and Curry and Webster (1999, pp. 162-163)], the entropy and equivalent potential temperature for an air-water vapor-liquid water mixture undergoing reversible ascent may be written

$$
\begin{aligned}
\frac{d s}{d t} & =c_{p} \frac{d \ln T}{d t}-R_{d} \frac{d \ln p_{d}}{d t}+\frac{d}{d t}\left(\frac{w_{\mathrm{vs}} \ell_{v}}{T}\right), \\
\theta_{\mathrm{es}} & \equiv T\left(\frac{p_{0}}{p_{d}}\right)^{R_{d} / c_{p}} \exp \left(\frac{w_{\mathrm{vs}} \ell_{v}}{c_{p} T}\right), \quad \text { and } \\
c_{p} & =\left(c_{\mathrm{pd}}+w_{t} c_{w}\right),
\end{aligned}
$$

where $T$ is the temperature, $R_{d}$ is the gas constant for dry air, $p_{d}$ is the partial pressure of dry air, $p_{0}$ is a constant reference pressure, $w_{\mathrm{vs}}$ is the saturation mixing ratio of water vapor with respect to dry air, $w_{t}$ is the total water (vapor plus liquid) mixing ratio, $\ell_{v}$ is the enthalpy of vaporization, $c_{\mathrm{pd}}$ is the heat capacity of dry air at constant pressure, and $c_{w}$ is the heat capacity of liquid water.

In a reversible process, specification of the state of the system requires three variables, one of which, such as $w_{t}$, determines the total amount of water. To reduce the required number of state variables to two, we consider pseudoadiabatic ascent, such that all liquid that forms immediately falls out of the air parcel. This choice is appropriate for the instantaneous diagnosis of largescale vertical motion, or for other applications where the total liquid water in the ascending air is unknown. Replacement of $w_{t}$ with $w_{\mathrm{vs}}\left(T, p_{d}\right)$ in (2.2), however, no longer allows the system to satisfy (2.1) exactly, since the coefficient $c_{p}$ in (2.2) is no longer constant. Further approximations, involving the assumption of negligible concentrations of water vapor, may be made to yield a system that formally satisfies (2.1):

$$
\begin{aligned}
\frac{d s}{d t} & =c_{\mathrm{pd}} \frac{d \ln T}{d t}-R_{d} \frac{d \ln p}{d t}+\frac{d}{d t}\left(\frac{w_{\mathrm{vs}} \ell_{v}}{T}\right) \text { and } \\
\theta_{\mathrm{es}} & \equiv T\left(\frac{p_{0}}{p}\right)^{R_{d} / c_{\mathrm{pd}}} \exp \left(\frac{w_{\mathrm{vs}} \ell_{v}}{c_{\mathrm{pd}} T}\right),
\end{aligned}
$$

where $p$ is the total pressure of the air parcel (and environment). So, because we assume pseudoadiabatic ascent, what follows involves an approximation either to the conservation of saturation equivalent potential temperature (2.1) or to the definition of saturation equivalent potential temperature [(2.3b) vs $(2.2 b)]$.

With the state of the system specified by any two state variables, we note the following identities:

$$
\begin{aligned}
\frac{d w_{\mathrm{vs}}\left(\theta_{\mathrm{es}}, p\right)}{d t} & =\left(\frac{\partial w_{\mathrm{vs}}}{\partial p}\right)_{\theta_{\mathrm{es}}} \frac{d p}{d t}+\left(\frac{\partial w_{\mathrm{vs}}}{\partial \theta_{\mathrm{es}}}\right)_{p} \frac{d \theta_{\mathrm{es}}}{d t} \\
& =\omega\left(\frac{\partial w_{\mathrm{vs}}}{\partial p}\right)_{\theta_{\mathrm{es}}}, \\
\frac{d \theta\left(\theta_{\mathrm{es}}, w_{\mathrm{vs}}\right)}{d t} & =\left(\frac{\partial \theta}{\partial w_{\mathrm{vs}}}\right)_{\theta_{\mathrm{es}}} \frac{d w_{\mathrm{vs}}}{d t}+\left(\frac{\partial \theta}{\partial \theta_{\mathrm{es}}}\right)_{w_{\mathrm{vs}}} \frac{d \theta_{\mathrm{es}}}{d t} \\
& =\left(\frac{\partial \theta}{\partial w_{\mathrm{vs}}}\right)_{\theta_{\mathrm{es}}} \frac{d w_{\mathrm{vs}}}{d t}, \quad \text { and } \\
{\left[\frac{\partial \theta\left(\theta_{\mathrm{es}}, p\right)}{\partial p}\right]_{x, y, t} } & =\left(\frac{\partial \theta}{\partial p}\right)_{\theta_{\mathrm{es}}}+\left(\frac{\partial \theta}{\partial \theta_{\mathrm{es}}}\right)_{p}\left(\frac{\partial \theta_{\mathrm{es}}}{\partial p}\right)_{x, y, t},
\end{aligned}
$$

where $\omega$ is the vertical motion in pressure coordinates and $\theta$ is the dry potential temperature. Equation (2.4a), which states that the rate of change in saturation mixing ratio of an air parcel is determined by its track along its pseudoadiabat, may be substituted into (2.4b) and the result rearranged to yield

$$
\left(\frac{\partial}{\partial t}+\mathbf{v}_{H} \cdot \boldsymbol{\nabla}\right) \theta+\omega\left[\frac{\partial \theta}{\partial p}-\left(\frac{\partial \theta}{\partial p}\right)_{\theta_{\mathrm{es}}}\right]=0
$$

where $\mathbf{v}_{H}$ is the vector horizontal wind. This expression explicitly illustrates the concept of effective stratification: latent heat release affects potential temperature as though the stratification were reduced by an amount equal to $(\partial \theta / \partial p)_{\theta_{\mathrm{es}}}$. Physically, the change in potential temperature caused by an upward parcel displacement is proportional to the departure of the potential temperature profile from a pseudoadiabat, just as for dry ascent the change in potential temperature is proportional to the departure of the potential temperature profile from a dry adiabat.

By substitution from $(2.4 \mathrm{c})$, 


$$
\left(\frac{\partial}{\partial t}+\mathbf{v}_{H} \cdot \boldsymbol{\nabla}\right) \theta+\omega\left[\left(\frac{\partial \theta}{\partial \theta_{\mathrm{es}}}\right)_{p} \frac{\partial \theta_{\mathrm{es}}}{\partial p}\right]=0 .
$$

Emanuel et al. (1987) showed that, for saturated conditions and assuming hydrostatic balance,

$$
\left(\frac{\partial s_{d}}{\partial s}\right)_{p}=\frac{c_{\mathrm{pd}}}{\alpha}\left(\frac{\partial T}{\partial p}\right)_{s}=\frac{\Gamma_{m}}{\Gamma_{d}},
$$

where $\alpha$ is the specific volume and $\Gamma_{d}$ and $\Gamma_{m}$ are the dry- and moist-adiabatic lapse rates, respectively. This result relies on a Maxwell's relation (e.g., Emanuel 1994 , p. 124) that is obtainable from the general relationship between entropy and enthalpy.

It follows from (2.7), using (2.1) and its analog for dry air, that

$$
\frac{\theta_{\mathrm{es}}}{\theta}\left(\frac{\partial \theta}{\partial \theta_{\mathrm{es}}}\right)_{p}=\frac{\Gamma_{m}}{\Gamma_{d}}
$$

so

$$
\left(\frac{\partial}{\partial t}+\mathbf{v}_{H} \cdot \boldsymbol{\nabla}\right) \theta+\omega\left(\frac{\Gamma_{m}}{\Gamma_{d}} \frac{\theta}{\theta_{\mathrm{es}}} \frac{\partial \theta_{\mathrm{es}}}{\partial p}\right)=0 .
$$

This "effective stratification" form of the thermodynamic equation for saturated ascent has been used, for example, by Emanuel et al. (1987). Analogous expressions can be obtained for height coordinates (Xu 1989) and for the thermodynamic equation cast in terms of temperature, all of which include the factor $\left(\Gamma_{m} / \Gamma_{d}\right)$. Durran and Klemp (1982) obtain a precise statement of the moist Brunt-Väisälä frequency that has a similar dependence on the moist- and dry-adiabatic lapse rates.

For practical applications, $\left(\Gamma_{m} / \Gamma_{d}\right)$ can be computed using any of several closed-form expressions given, for example, by Iribarne and Godson (1981, pp. 180-183); an approximate form appropriate for pseudoadiabats is

$$
\frac{\Gamma_{m}}{\Gamma_{d}} \approx \frac{1+\frac{\varepsilon \ell_{v} e_{s}}{R_{d} T p}}{1+\frac{\varepsilon^{2} \ell_{v}^{2} e_{s}}{c_{\mathrm{pd}} R_{d} T^{2} p}},
$$

where $\varepsilon=R_{d} / R_{v}=0.622$ and $e_{s}$ is the saturation vapor pressure.

\section{An incorrect derivation}

Here we discuss the derivation that appears in Holton (1992, p. 389) and leads to a sometimes-used, but incorrect, version of (2.9). We follow the notation and coordinate system of section 2. Beginning with an approximate form of the first law, under pseudoadiabatic ascent [compare with (2.3a)],

$$
\frac{d \ln \theta_{\mathrm{es}}}{d t}=\frac{d \ln \theta}{d t}+\left(\frac{\ell_{v}}{c_{\mathrm{pd}} T}\right) \frac{d w_{\mathrm{vs}}}{d t}=0,
$$

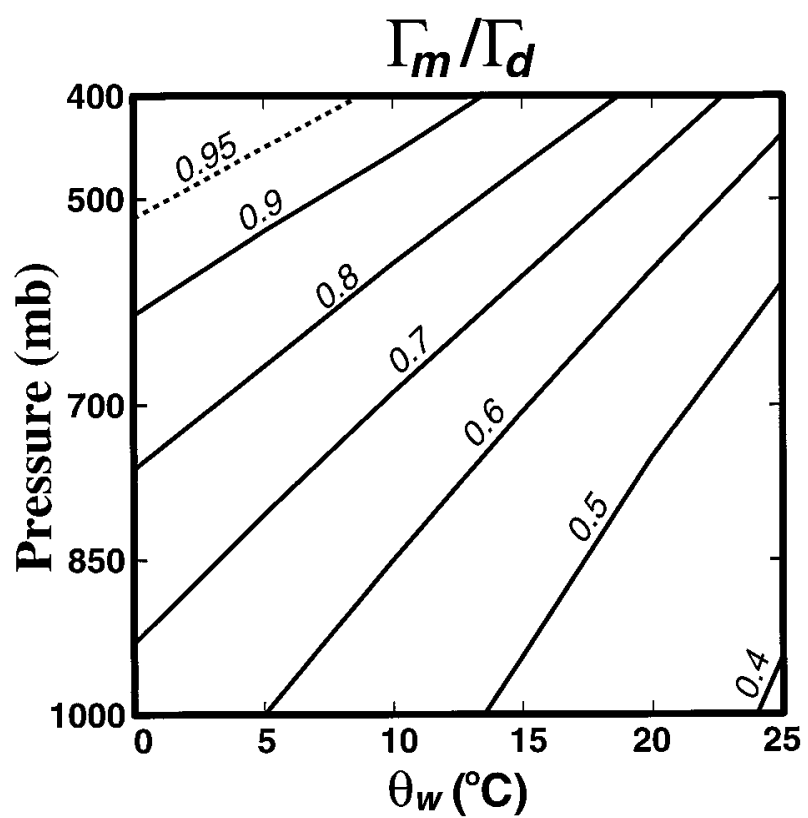

FIG. 1. The magnitude of the correction factor $\left(\Gamma_{m} / \Gamma_{d}\right)$ for typical values of pressure and wet-bulb potential temperature.

Holton notes that changes in $w_{\mathrm{vs}}$ following the motion are associated primarily with the ascent itself, so that

$$
\frac{d w_{\mathrm{vs}}}{d t} \approx \omega \frac{\partial w_{\mathrm{vs}}}{\partial p},
$$

and (3.1) may be written

$$
\left(\frac{\partial}{\partial t}+\mathbf{v}_{H} \cdot \boldsymbol{\nabla}\right) \ln \theta+\omega\left(\frac{\partial \ln \theta}{\partial p}+\frac{\ell_{v}}{c_{\mathrm{pd}} T} \frac{\partial w_{\mathrm{vs}}}{\partial p}\right) \approx 0 .
$$

Neglecting changes in $l_{v}$ and $T$ in (2.3b) compared to changes in $w_{\mathrm{vs}}$ yields

$$
\frac{\partial \ln \theta_{\mathrm{es}}}{\partial p}=\frac{\partial \ln \theta}{\partial p}+\frac{\ell_{v}}{c_{\mathrm{pd}} T} \frac{\partial w_{\mathrm{vs}}}{\partial p} .
$$

It thus follows for moist ascent that

$$
\left(\frac{\partial}{\partial t}+\mathbf{v}_{H} \cdot \boldsymbol{\nabla}\right) \theta+\omega\left(\frac{\theta}{\theta_{\mathrm{es}}} \frac{\partial \theta_{\mathrm{es}}}{\partial p}\right) \approx 0 .
$$

This expression, which seems to make physical sense because it involves a simple replacement of $\left(\theta / \theta_{\mathrm{es}}\right)\left(\partial \theta_{\mathrm{es}} / \partial p\right)$ for $\partial \theta / \partial p$ in the thermodynamic equation, differs from (2.9) by the factor $\left(\Gamma_{m} / \Gamma_{d}\right)$. This factor lies between 0 and 1 and is significantly different from 1 at ordinary temperatures and pressures (Fig. 1). This incorrect form of effective stratification also appears in Carlson (1991, p. 70) and has been used, for example, by Hirschberg and Fritsch (1991).

The origin of the discrepancy may be seen by comparing (3.2) and (2.4a). In (2.4a), the variation of $w_{v s}$ following an air parcel is determined by the rate of change of $w_{\mathrm{vs}}$ along the pseudoadiabat that the air parcel 
follows. In (3.2), the variation of $w_{\mathrm{vs}}$ following an air parcel is incorrectly specified by the environmental variation of $w_{\mathrm{vs}}$ with respect to pressure. The discrepancy appears as a correction factor because the difference between the environmental variation of $w_{\mathrm{vs}}$ and the variation of $w_{\mathrm{vs}}$ along a pseudoadiabat is itself proportional to the departure of the environment from a pseudoadiabat, as is seen by application of the chain rule to $\partial w_{\mathrm{vs}} / \partial p$ :

$$
\frac{\partial w_{\mathrm{vs}}}{\partial p}-\left(\frac{\partial w_{\mathrm{vs}}}{\partial p}\right)_{\theta_{\mathrm{es}}}=\left(\frac{\partial w_{\mathrm{vs}}}{\partial \theta_{\mathrm{es}}}\right)_{p} \frac{\partial \theta_{\mathrm{es}}}{\partial p} .
$$

An expression identical to (2.9) ultimately is obtained from (3.3) if (2.4a) replaces (3.2).

\section{Discussion}

The consequence of the erroneous (3.5) is an overestimate of the effective stratification when the atmosphere is stable to moist ascent. This overestimate is so large that, as can be seen from (3.3), the effect of latent heat release is neglected completely when the environmental saturation mixing ratio is constant with height. When the atmosphere is even more stable, such as when a temperature inversion is present, the incorrect expressions diagnose "latent cooling" due to condensation, such that ascending air cools at a rate greater than the dry-adiabatic lapse rate!

Although (3.5) produces erroneous results, we are not aware of any published conclusions invalidated by its use. In Hirschberg and Fritsch (1991), for example, the erroneous diagnosis of vertical motion using (3.5) was effectively canceled by an analogous error in the estimate of height tendencies, so the results of that paper remain valid.

In summary, the effective stratification experienced by the atmosphere during moist-adiabatic ascent is given approximately by (2.9). Equation (3.5) is not merely an approximation; it is erroneous and leads to physically unrealistic results.

Acknowledgments. This research was supported by the National Science Foundation through Grants ATM8805550 and ATM-9818088 to the University at Albany and ATM-9553284 to Texas A\&M University.

\section{REFERENCES}

Bohren, C. F., and B. A. Albrecht, 1998: Atmospheric Thermodynamics. Oxford University Press, $402 \mathrm{pp}$.

Carlson, T. N., 1991: Mid-Latitude Weather Systems. HarperCollins Academic, 507 pp.

Curry, J. A., and P. J. Webster, 1999: Thermodynamics of Atmospheres and Oceans. Academic Press, $471 \mathrm{pp}$.

Davis, C. A., E. Donall Grell, and M. A. Shapiro, 1996: The balanced dynamical nature of a rapidly intensifying oceanic cyclone. Mon. Wea. Rev., 124, 3-26.

Durran, D. R., and J. B. Klemp, 1982: On the effects of moisture on the Brunt-Väisälä frequency. J. Atmos. Sci., 39, 2152-2158.

Emanuel, K. A., 1985: Frontal circulations in the presence of small moist symmetric stability. J. Atmos. Sci., 42, 1062-1071.

_, 1994: Atmospheric Convection. Oxford University Press, 580 pp.

_- M. Fantini, and A. J. Thorpe, 1987: Baroclinic instability in an environment of small stability to slantwise moist convection. Part I: Two-dimensional models. J. Atmos. Sci., 44, 1559-1573.

Hauf, T., and H. Höller, 1987: Entropy and potential temperature. $J$. Atmos. Sci., 44, 2887-2901.

Hirschberg, P. A., and J. M. Fritsch, 1991: Tropopause undulations and the development of extratropical cyclones. Part II: Diagnostic analysis and conceptual model. Mon. Wea. Rev., 119, 518550.

Holton, J. R., 1992: An Introduction to Dynamic Meteorology. 3d ed. Academic Press, 511 pp.

Iribarne, J. V., and W. L. Godson, 1981: Atmospheric Thermodynamics. 2d ed. D. Reidel, 259 pp.

Kuo, Y.-H., M. A. Shapiro, and E. G. Donall, 1991: The interaction between baroclinic and diabatic processes in a numerical simulation of a rapidly intensifying extratropical marine cyclone. Mon. Wea. Rev., 119, 368-384.

Lagouvardos, K., Y. Lemaitre, and G. Scialom, 1993: Importance of diabatic processes on ageostrophic circulations observed during the FRONTS 87 experiment. Quart. J. Roy. Meteor. Soc., 119, $1321-1345$.

Orlanski, I., B. Ross, L. Polinsky, and R. Shaginaw, 1985: Advances in the theory of atmospheric fronts. Advances in Geophysics, Vol. 28B, Academic Press, 223-252.

Pauley, P. M., and S. J. Nieman, 1992: A comparison of quasigeostrophic and nonquasigeostrophic vertical motions for a modelsimulated rapidly intensifying marine extratropical cyclone. Mon. Wea. Rev., 120, 1108-1134.

Schultz, D. M., and W. J. Steenburgh, 1999: The formation of a forward-tilting cold front with multiple cloud bands during Superstorm 1993. Mon. Wea. Rev., 127, 1108-1124.

Whitaker, J. S., and C. A. Davis, 1994: Cyclogenesis in a saturated environment. J. Atmos. Sci., 51, 889-907.

Xu, Q., 1989: Extended Sawyer-Eliassen equation for frontal circulations in the presence of small viscous moist symmetric stability. J. Atmos. Sci., 46, 2671-2683. 